\title{
Design optimization of field-plate assisted RESURF devices
}

\author{
B.K. Boksteen, A. Ferrara, A. Heringa ${ }^{2}$, P.G. Steeneken ${ }^{1}$, G.E.J. Koops ${ }^{2}$, R.J.E. Hueting \\ MESA+ Institute for Nanotechnology, University of Twente, 7500AE, Enschede, The Netherlands \\ NXP Semiconductors: $\quad{ }^{1}$ Eindhoven, The Netherlands $\quad{ }^{2}$ Leuven, Belgium \\ Tel./Fax: +31 53489 2645/6920Ｅmail: b.k.boksteen@utwente.nl
}

\begin{abstract}
A mathematical model for optimizing the 2-D potential distribution in the drift region of field-plate (FP)assisted RESURF devices (Fig. 1) is presented. The proposed model extends earlier work [1-2] by including top-bottom dielectric asymmetry (typical in SOI devices [3]), non-zero field plate potentials $V_{F P}$ and grading of design parameters other than drift region doping. This generally-applicable, TCAD-verified [4], model provides a guideline for optimizing the drain extension in a wide range of FP-assisted RESURF devices.
\end{abstract}

\section{INTRODUCTION}

The RESURF (Reduced SURface Field [5]) effect is commonly used to improve the specific on-resistance vs. breakdown voltage $\left(R_{\mathrm{on}} A-B V\right)$ trade-off in high-voltage (HV) transistors [6]. RESURF optimization aims to achieve a constant horizontal field at breakdown using various methods: pn-junctions [5], super-junctions [7], field-plates [8] or a combination [9]. FP-assisted RESURF can be realized in both SOI [8] and trench-MOS [10] technology by tailoring one of the following design parameters: (Fig. 1a) drift region doping $N_{\mathrm{D}}$ [2, 11], (Fig. 1b) dielectric layer permittivity $\varepsilon_{\mathrm{d}}$ [12], (Fig. 1c) dielectric layer thickness $t_{\mathrm{d}}$ [13], (Fig. 1d) semiconductor layer thickness $t_{\mathrm{s}}[14]$ and (Fig. 1e) FPpotential $V_{\mathrm{FP}}[15,16]$. In this work, a complete mathematical model is presented that allows $R_{\mathrm{on}} A-B V$ optimization for all above mentioned FP-RESURF devices.

The paper is outlined as follows: Section II presents a modular look at the mathematical model and its TCAD verification; Section III focuses on its application and attainable $R_{\mathrm{on}} A-B V$ trade-off, while in Section IV conclusions are drawn.

\section{FP-ASSISTED RESURF MODEL}

The model proposed in this paper extends the work by $\mathrm{S}$. Merchant [2] to a broader application range, while achieving deeper physical insight into the trade-offs related to $R_{\mathrm{on}} A-B V$ optimization of FP-assisted RESURF devices. For this purpose, a more general model is presented describing the optimal RESURF electric fields and potential distributions in drain extensions using any substrate/dielectric combination or (2-D) symmetry (Fig. 1).

\section{A. Constant Lateral Field Condition}

The general description of the field distribution in the depleted drain extension is given by the 2-D Poisson equation (assuming only a lateral variation in drift doping $N_{\mathrm{D}}(x)$ and semiconductor permittivity $\left.\varepsilon_{\mathrm{s}}\right)$ :

$$
\frac{d E_{x}(x, y)}{d x}+\frac{d E_{y}(x, y)}{d y}=\frac{q N_{\mathrm{D}}(x)}{\varepsilon_{\mathrm{s}}}
$$

(a-b)

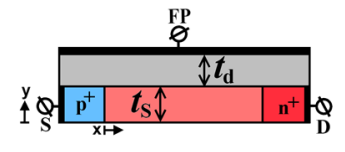

(a)

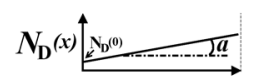

(b)

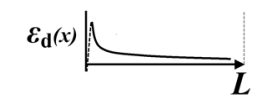

(c)

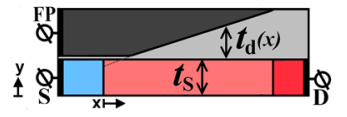

(d)

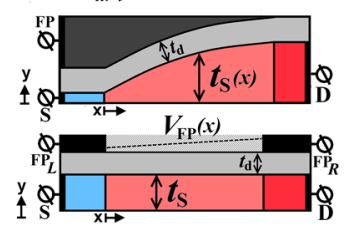

Fig. 1: Breakdown voltage $(B V)$ optimization methods for FP-assisted RESURF devices. Optimal structures shown for: a) graded $N_{\mathrm{D}}$; b) graded $\varepsilon_{\mathrm{d}}$; c) graded $\left.t_{\mathrm{d}} ; \mathbf{d}\right)$ graded $t_{\mathrm{s}} ; \mathbf{e}$ ) graded $V_{\mathrm{FP}}$. The methods shown are also applicable to vertical devices.

Since optimal RESURF design requires a constant horizontal field $E_{x}=-B V / L$ at breakdown [2], Eq. 1 can be solved for the 2-D potential distribution giving $\psi(x, y)=\frac{B V}{L} x+V(x, y)$ with $V(x, y)$ obtained by integrating Eq. 1 and using $d E_{x} / d x=0$. This yields the general expression:

$$
\psi(x, y)=\frac{B V}{L} x-\frac{q N_{\mathrm{D}}(x)}{2 \varepsilon_{\mathrm{s}}} y^{2}
$$

It can be shown (Fig. 2) that various drain extensions (singlesided (SS), double-sided asymmetrical (ASYM) and symmetrical (SYM)) can be modeled as a symmetrical fieldplate/semiconductor structure with equivalent MOS depletion thickness $t_{\mathrm{eq}}(x)$. For a SS device, $t_{\mathrm{eq}}=\sqrt{2 t_{\mathrm{s}}\left(\frac{t_{\mathrm{s}}}{2}+\frac{\varepsilon_{\mathrm{s}}}{\varepsilon_{\mathrm{d}}} t_{\mathrm{d}}\right)}$ and is related to the $\lambda$-parameter introduced in [2] (see Eq. 6a) using the relation $t_{\mathrm{eq}}=\sqrt{2} \lambda$. Imposing the boundary condition $\psi\left(x, t_{\mathrm{eq}}(x)\right)=V_{\mathrm{FP}}(x)$ to Eq. 2 and rearranging the terms yields the general constant lateral field condition:

$$
\frac{\varepsilon_{\mathrm{s}}\left[\frac{B V}{L} x-V_{\mathrm{FP}}(x)\right]}{q N_{\mathrm{D}}(x) \frac{t_{\mathrm{eq}}^{2}(x)}{2}}=1
$$

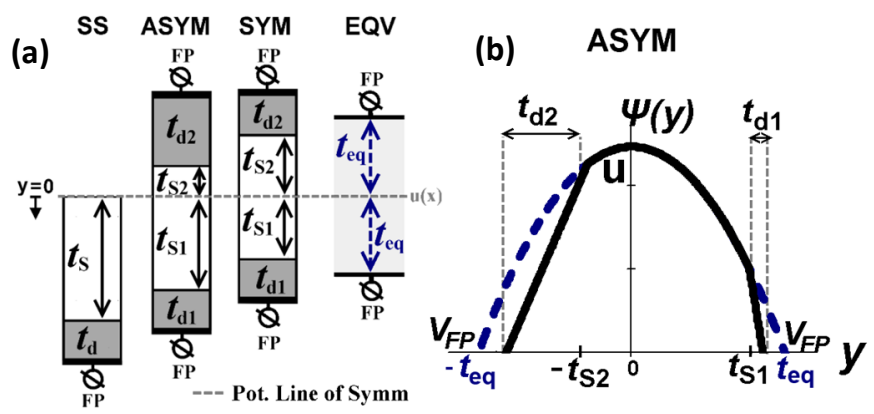

Fig. 2: a) Cut along the $y$-direction of cross sections shown in Fig. 1 for SS, $\overline{\mathrm{ASYM}}$ and SYM devices and the equivalent structure. Dark gray: dielectric, white/light gray: semiconductor b) Potential distribution $\psi(y)$ for ASYM devices including representation of $t_{\text {eq }}$. 
$\underbrace{\frac{\varepsilon_{\mathrm{S}}\left(\frac{B V}{L} x-V_{\mathrm{FP}}\right)}{q N_{\mathrm{D}} \lambda^{2}}=1}_{\text {(4) }} \Rightarrow\left\{\begin{array}{l}N_{\mathrm{D}}(x)=\frac{\varepsilon_{\mathrm{s}}}{q \lambda^{2}}\left(\frac{B V}{L} x-V_{\mathrm{FP}}\right) \\ \lambda^{2}(x)=\frac{\varepsilon_{\mathrm{s}}}{q N_{\mathrm{D}}}\left(\frac{B V}{L} x-V_{\mathrm{FP}}\right) \\ V_{\mathrm{FP}}(x)=\frac{B V}{L} x-\frac{q N_{\mathrm{D}}}{\varepsilon_{\mathrm{s}}} \lambda^{2}\end{array}\right.$

Rewriting Eq. 3 in terms of $\lambda$ leads to Eq. 4 , which describes how the different design parameters can be tailored (Fig. 1) in order to achieve optimal RESURF by means of: (a) graded doping (Eq. 4a and Fig. 1a), (b) " $\lambda$-RESURF" achieved through graded dielectric constant (Eq. $4 \mathrm{i}$ and Fig. 1b) or graded layer $\left(t_{\mathrm{d}}\right.$ or $\left.t_{\mathrm{s}}\right)$ thicknesses (Figs. 1c-d and Eqs. 4ii-iii), and (c) FP-potential RESURF (Eq. 4c and Fig. 1e). Optimal RESURF can also be obtained using a combination of graded profiles as long as Eq. 4 is fulfilled. Although the graded profiles from Eq. 4 are not always exact solutions of the Poisson equation, good agreement between the model and TCAD simulations is achieved.

From each of the boundary conditions corresponding to the different vertical device symmetries (SS, ASYM and SYM), a relation for $t_{\mathrm{eq}}$ and therefore $\lambda$ can be derived:

$$
\begin{gathered}
\lambda=\left\{\begin{array}{l}
\sqrt{t_{\mathrm{s}}\left(\frac{t_{\mathrm{s}}}{2}+\frac{\varepsilon_{\mathrm{s}}}{\varepsilon_{\mathrm{d}}} t_{\mathrm{d}}\right)} \Rightarrow \mathrm{SS} \\
\sqrt{t_{\mathrm{s} 1,2}\left(\frac{t_{\mathrm{s} 1,2}}{2}+\frac{\varepsilon_{\mathrm{s}}}{\varepsilon_{\mathrm{d} 1,2}} t_{\mathrm{d} 1,2)}\right)} \Rightarrow \mathrm{ASYM} \\
\sqrt{\frac{t_{\mathrm{s}}}{2}\left(\frac{t_{\mathrm{s}}}{4}+\frac{\varepsilon_{\mathrm{s}}}{\varepsilon_{\mathrm{d}}} t_{\mathrm{d}}\right)} \Rightarrow \mathrm{SYM}
\end{array}\right. \\
t_{\mathrm{s} 1,2}=\frac{\frac{t_{\mathrm{s}}}{2}\left(t_{\mathrm{s}}+2 \frac{\varepsilon_{\mathrm{s}}}{\varepsilon_{\mathrm{d} 2,1}} t_{\mathrm{d} 2,1)}\right.}{t_{\mathrm{s}}+\frac{\varepsilon_{\mathrm{s}}}{\varepsilon_{\mathrm{d} 1}} t_{\mathrm{d} 1}+\frac{\varepsilon_{\mathrm{s}}}{\varepsilon_{\mathrm{d} 2}} t_{\mathrm{d} 2}}
\end{gathered}
$$

Equations 4-6 thus provide a guideline for RESURF optimization across a wide range of drain extensions types. However, they will only lead to optimal RESURF if the device breakdown voltage is limited by the lateral field $E_{x}$, as discussed in following section.

\section{B. Breakdown}

Breakdown in 2-D structures can occur in three possible ways: lateral $\left(E_{x}\right)$ breakdown, vertical $\left(E_{y}\right)$ breakdown and P-body $/ \mathrm{N}^{-}$ -drain junction breakdown. The breakdown voltage $B V$ of the device is determined by the lowest of each of the three contributions. In this section, the breakdown mechanisms are analyzed by: 1) solving Eq. 7 (obtained using [2]) for $u(x)$, representing the potential along the symmetry line $y=0(u(x)=$ $\psi(x, 0)) ; 2$ ) deriving the field distributions from Eq. 8; and 3) calculating the impact ionization (II) integrals using Eqs. 9-10. The results are then compared with TCAD simulations.

$$
\begin{gathered}
\frac{d^{2} u(x)}{d x^{2}}-\frac{u(x)-V_{\mathrm{FP}}(x)}{\lambda^{2}(x)}=-\frac{q N_{\mathrm{D}}(x)}{\varepsilon_{\mathrm{S}}} \\
E_{x}(x, 0)=-u^{\prime}(x) \\
E_{y}(x, y)=\frac{u(x)-V_{\mathrm{FP}}(x)}{\lambda^{2}(x)} y
\end{gathered}
$$

$E_{x}$ breakdown: (Fig. 3) occurs when the II-integral ( $\alpha_{x}$ (in Si), Eq. 9a) along the symmetry line in the drift region $(y=0$, path 1

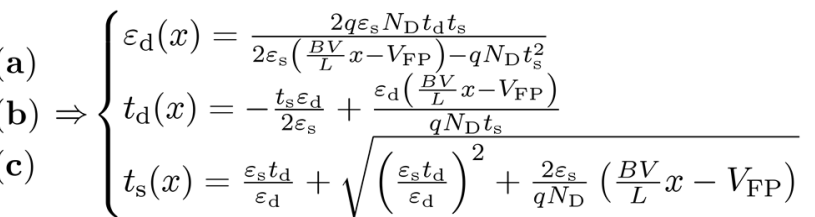

Fig. 4a) reaches 1 . Since for $V_{\mathrm{DS}}=B V$ the longitudinal field $E_{x}=B V / L$ at $y=0$, the integration of $\alpha_{x}$ simply returns the optimal $B V$ vs. drift length $(L)$ relation (Eq. $9 \mathrm{~b})$.

$\alpha_{x}=\int_{\text {Path } 1} a_{n} \cdot \exp \left(\frac{-b_{n}}{E_{x}(x, 0)}\right) \mathrm{d} x \mathbf{( 9 a )}, B V_{\text {ideal }}=\frac{b_{n} L}{\ln \left(a_{n} L\right)}$

Using the silicon coefficients $a_{\mathrm{n}}=7.03 \cdot 10^{5} \mathrm{~cm}^{-1}, b_{\mathrm{n}}=1.47 \cdot 10^{6}$ $\mathrm{Vcm}^{-1}[8]$ and the lateral field description (Eq. 8), the results obtained from the model and TCAD are in good agreement for the whole $V_{\mathrm{DS}}$ range and across multiple (optimal and nonoptimal) gradient values (Fig. 3).

(a)

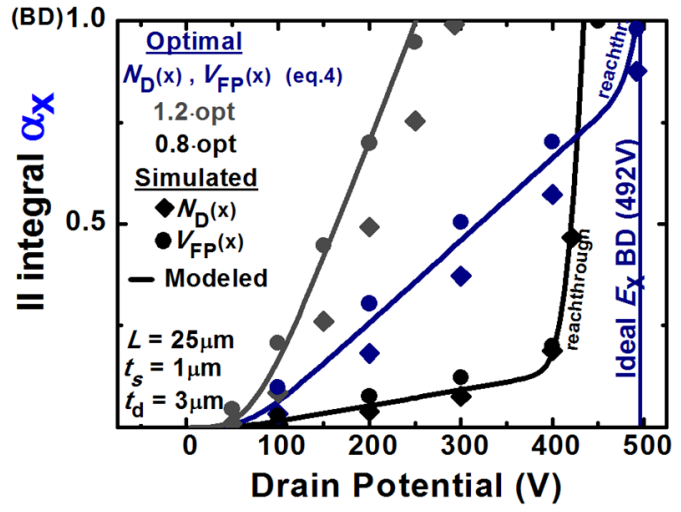

(b)

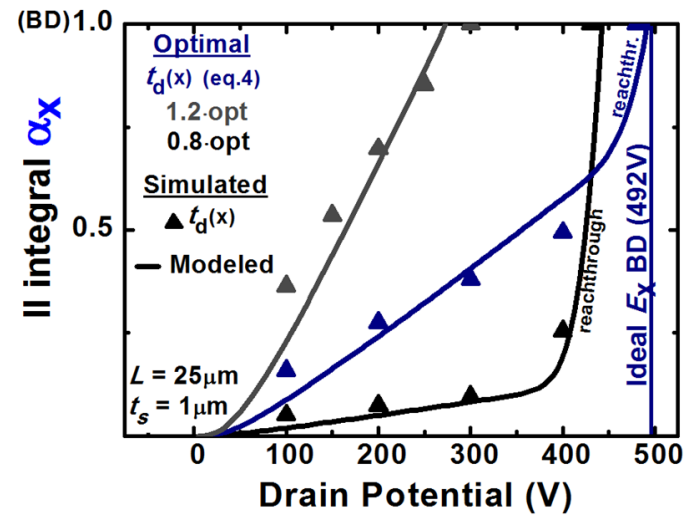

Fig.3: a) TCAD simulated and modeled $E_{x}$-ionization integrals ( $E_{x}$-II) for SYM graded $-N_{\mathrm{D}}$ and graded- $V_{\mathrm{FP}}$ RESURF in three different cases: optimal (opt) graded-RESURF slope (for max. $E_{x}-B V$, Eq. 4$)$, reduced grading $(0.8 \cdot \mathrm{opt})$ and increased grading $(1.2 \cdot \mathrm{opt})$. b) As in a) but for graded- $t_{\mathrm{d}}$. For all simulations a $\mathrm{Si} / \mathrm{SiO}_{2}$ structure is assumed with a fixed $\varepsilon_{\mathrm{s}} / \varepsilon_{\mathrm{d}}$ ratio of $\sim 3$.

$\underline{E}_{y}$ breakdown: (Figs. 4-5) The $E_{y}$ II-integral (Eq. 10) is calculated along the longest path (amongst $\mathrm{t}_{\mathrm{s} 1}$ and $\mathrm{t}_{\mathrm{s} 2}$ ) at the edge of the depletion region $\left(x=W\left(V_{\mathrm{DS}}\right)\right.$, path 2 in Fig. $\left.4 \mathrm{a}\right)$, where $E_{y}$ is maximum. It is worth mentioning that the solution of Eq. 10 is not analytical. In addition, a transcendental equation $[2,17]$ needs to be solved for determining $W\left(V_{\mathrm{DS}}\right)$.

$$
\alpha_{y}=\int_{P a t h 2} a_{n} \cdot \exp \left(\frac{-b_{n}}{E_{y}\left(W\left(V_{D S}\right), y\right)}\right) \mathrm{d} y
$$




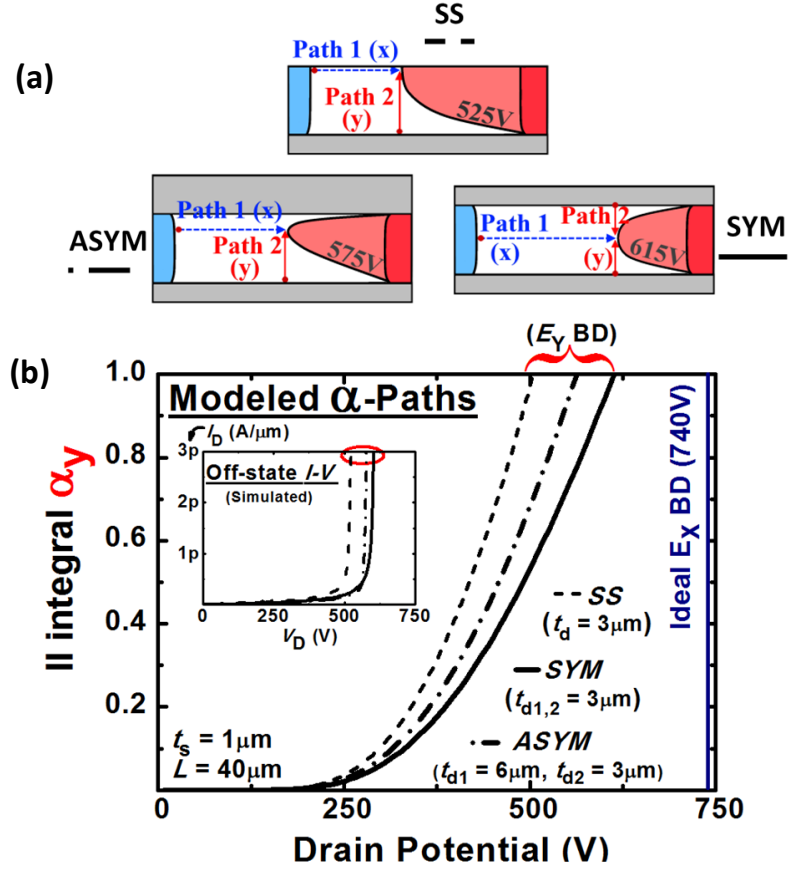

Fig.4: a) Highest electron impact ionization (highest $e^{-}$-II) paths [17] for $B V$ modeling in SS, ASYM and SYM structures. b) $E_{y}$-II integrals calculated for SYM, ASYM and SS devices showing premature vertical breakdown as the $y$-path length (Path 2) increases. Inset: matching simulated off-state $I-V$ in the three cases.

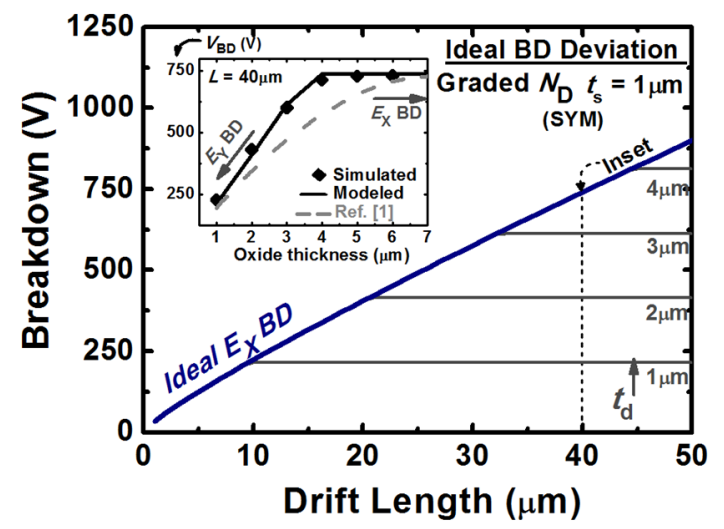

Fig.5: $B V$ vs. drift length $L$ for graded- $N_{\mathrm{D}}$ RESURF, showing the limitations on the choice of $t_{\mathrm{d}}$ imposed by $E_{y}$-breakdown. Inset shows a comparison between simulated and modeled $B V$ for $L=40 \mu \mathrm{m}$.

Modeled and TCAD verified $E_{y}$ breakdown limited cases are compared in Fig. 4b (for increasing vertical path 2 length) and Fig. 5 (for increasing $E_{y}$ field peak). Breakdown occurs when $\max \left(\alpha_{x}, \alpha_{y}\right)=1$, as verified by TCAD (inset of Fig. 5 ).

Junction breakdown: (Fig. 6) A high initial doping $N_{0}\left(N_{\mathrm{D}}(x=0)\right)$ can lead to an increased $E_{x}$ field peak (at $x=0$, Fig. 6a) causing premature junction breakdown. Its occurrence was first described by Appels and Vaes in [5] resulting in the general max. junction dose rule $t_{s} \cdot N_{D}(0)=2 \cdot 10^{12}$. The TCAD results in Fig. 6 show how junction breakdown can limit the ideal device performance of $t_{\mathrm{d}}$-graded RESURF extensions.
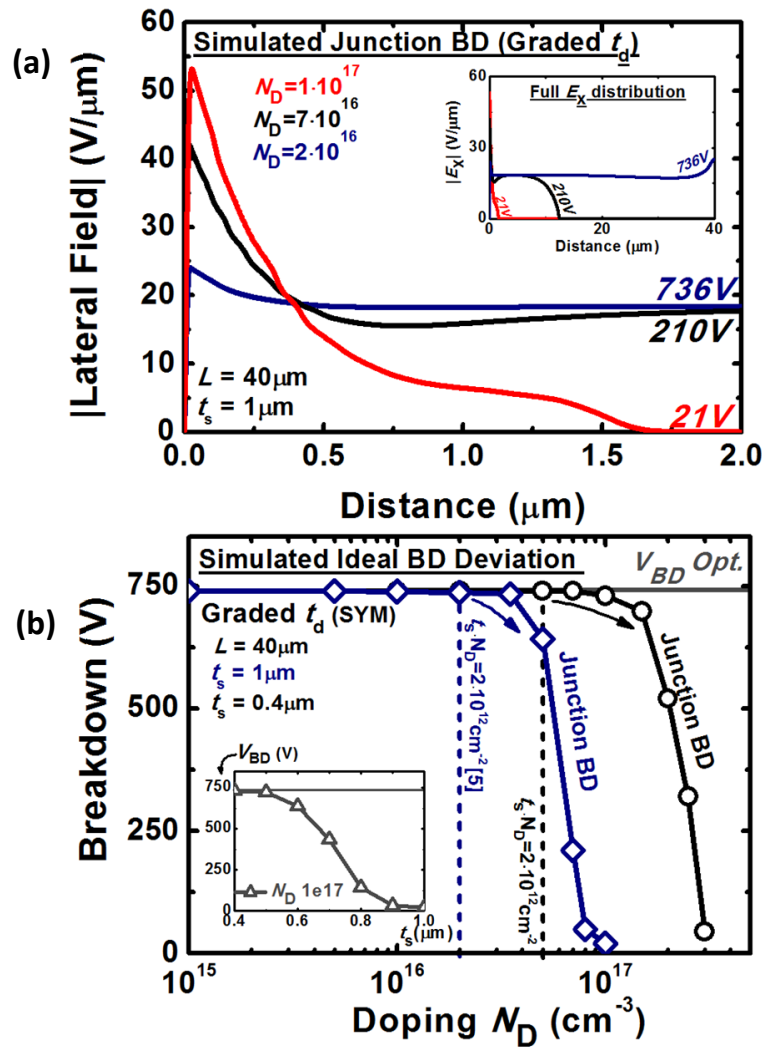

Fig.6: a) Junction breakdown for $t_{\mathrm{d}}$-graded RESURF. As the uniform doping concentration $N_{\mathrm{D}}$ is increased, the higher $E_{x}$-field peak at the $p n$-junction interface leads to premature 1-D breakdown preventing full $E_{x}$-expansion in the drift region. b) $B V$ vs. $N_{\mathrm{D}}$ for different thicknesses $t_{\mathrm{s}}$ showing how the onset of junction breakdown is shifted towards higher $N_{\mathrm{D}}$ when reducing $t_{\mathrm{s}}$ (see also inset).

\section{Field-plate potential}

Obtaining a constant $E_{x}$ is in principle not always possible with grounded field-plates $\left(V_{\mathrm{FP}}=0 \mathrm{~V}\right)$, because Eq. 4 predicts unrealistic (e.g. negative) values for device parameters at $x=0$. Equation 12 shows how to tailor the $V_{\mathrm{FP}}$-value to achieve a constant $E_{x}$ for different types of FP-RESURF. Vertical and junction breakdown are avoided by tuning the non-graded parameters (Fig. 7a-c).

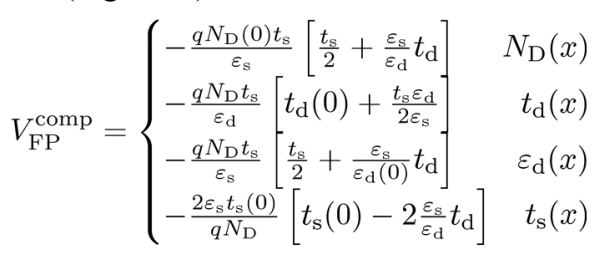

\section{ApPLiCATION GUIDELINE AND Results}

Using the proposed model, the following guideline for optimizing FP-assisted RESURF design can be derived: 1) first determine the drift-region length $(L)$ for the desired ideal $B V$ from Eq. 9b; then 2) optimize the lateral (x) $B V$ (Fig. 3) by grading one of the design parameters according to Eq. 4; finally 3) tailor the other device parameters such that: a) the device is not subjected to vertical breakdown (Figs. 4-5) or 
(a)

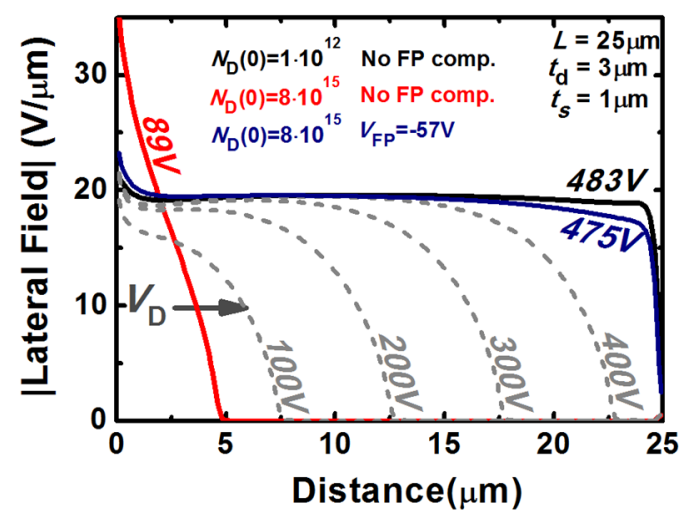

(c)

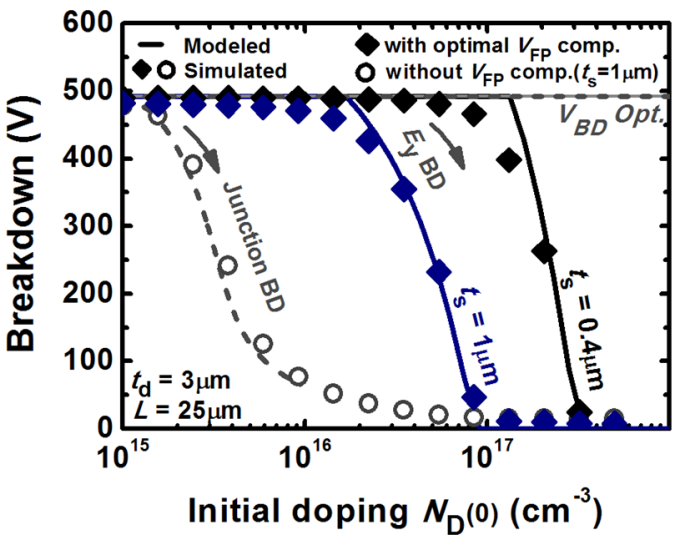

(b)

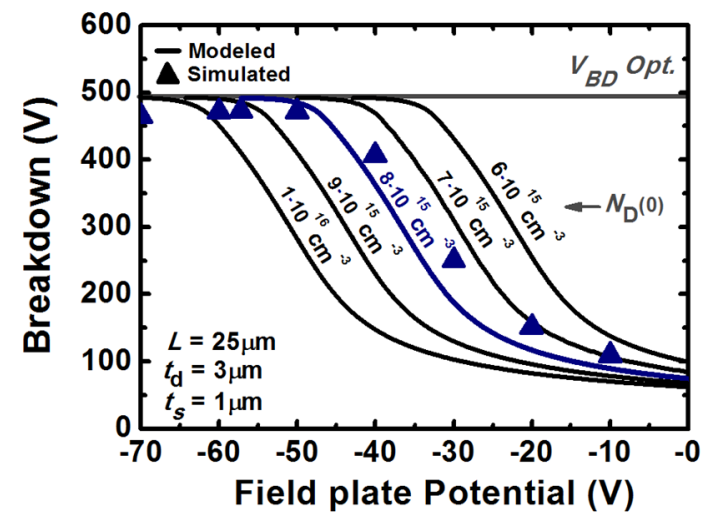

(d)

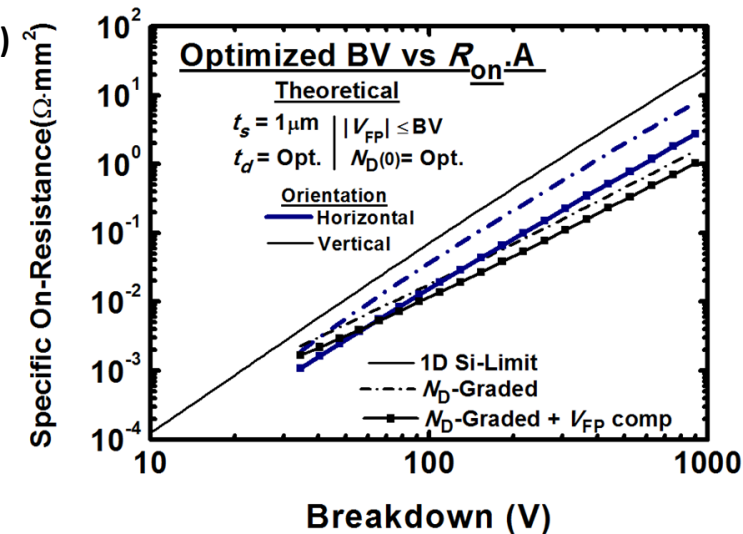

Fig. 7: a) Simulated $E_{x}$-fields at breakdown for graded- $N_{\mathrm{D}}$ RESURF with and without $V_{\mathrm{FP}}$-compensation showing optimal $E_{x}$-breakdown (Eq. 7) when the right $V_{\mathrm{FP}}$ comp value (Eq. 10) is applied. b) Simulated and modeled $E_{\mathrm{x}}-B V$ Vs. $V_{\mathrm{FP}}$ for different values of the initial doping $N_{\mathrm{D}}(0)$. c) Simulated and modeled $B V$ vs. $N_{\mathrm{D}}(0)$

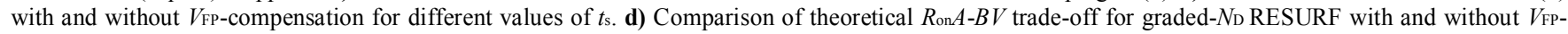
compensation for both horizontal and vertical devices. For $t_{\mathrm{s}}=1 \mu \mathrm{m}$ (SYM device) and optimal $N_{\mathrm{D}}$ (x) profile (Eq. $\left.4 \mathrm{a}\right)$, the other design parameters $\left(t_{\mathrm{d}}\right.$ and $N_{\mathrm{D}}(0)$ ) are selected to achieve minimum $R_{\text {on }} A$ for ideal $B V$.

junction breakdown (Fig. 6); and b) the specific on-resistance $R_{\mathrm{on}} A$ is minimized (Fig. 7d).

\section{Specific on-resistance $\left(R_{\mathrm{on}} A\right)$ optimization}

A negatively-biased $V_{\mathrm{FP}}$ in off-state can be used to reduce the specific $R_{\mathrm{on}} A$ of the device without affecting the $B V$ (Fig. 7ac). This is possible because a negative FP-potential acts on the depleted charge distribution as an equivalent doping of opposite polarity. Therefore, an arbitrarily-high drift doping can in principle be used if properly compensated by a $V_{\mathrm{FP}}<0$, as suggested by Eq. 12 for $N_{\mathrm{D}}(\mathrm{x})$. In practice, extreme values of $N_{\mathrm{D}}(\mathrm{x})$ might lead to premature vertical breakdown (Fig. 7c), thus limiting the minimum achievable resistivity in the drift region. Using a numerical optimization routine, the limit value of the drift doping $N_{\mathrm{D}}(x)$ for achieving minimum $R_{\text {on }} A$ while preventing vertical breakdown can be determined. Performing such optimization on a graded- $N_{\mathrm{D}}$ device (Fig. 7d) shows that a theoretical $R_{\mathrm{on}} A-B V$ improvement (by a factor $\sim 5$ for lateral and $\sim 2$ for vertical devices) can be achieved when using negatively-biased $V_{\mathrm{FP}}$ compared to its grounded $V_{\mathrm{FP}}$ counterpart.

\section{CONCLUSION}

A mathematical model describing field and potential distributions in different configurations of FP-assisted
RESURF devices has been presented and verified by TCAD simulations. Using the proposed model, an optimal $R_{\text {on }} A-B V$ trade-off can be achieved for both horizontal and vertical devices.

\section{ACKNOWLEDGMENT}

This work is a part of the Dutch Point-One program and is supported financially by Agentschap NL, an agency of the Dutch Ministry of Economic Affairs.

\section{REFERENCES}

[1] S. Merchant et al., Proc. ISPSD'91, pp. 31-35 (1991)

[2] S. Merchant, TED, 46 (6), pp. 1264-1267 (1999)

[3] P. Wessels et al., SSE 51, pp. 195-211 (2007)

[4] Silvaco's ATLAS (Version: 5.16.3.R)

[5] J.A. Appels \& H.M.J. Vaes, IEDM'79, pp. 238-241 (1979)

[6] A.W. Ludikhuize, Proc. ISPSD’00, pp. 11-18 (2000)

[7] T. Fujihira, Jpn. J. Appl. Phys. 36 (10) Part 1, pp. 6254-6262 (1997)

[8] S. Merchant et al., Proc. ISPSD'93, pp.124-128 (1993)

[9] E.K. Choi et al., MICROELECTR J 34, pp. 683-686 (2003)

[10] S. Mahalingam \& B.J. Baliga, Proc. ISPSD’98, pp. 187-190 (1998)

[11] J. He et al., SST 17, pp. 721-728 (2002)

[12] S. Luo et al., SSE 51, pp. 493-499 (2007)

[13] Y. Chen et al., TPE 22 (4), pp. 1303-1310 (2007)

[14] Q. Xu et al., IWIEE'12, pp. 668-672 (2012)

[15] G.A.M. Hurkx \& R. van Dalen, EP1169738A1 (2002)

[16] S. Schwantes et al., TED 52 (7), pp. 1649-1655 (2005)

[17] B.K. Boksteen et al., Proc. IEDM'12 (2012) 